\title{
Homogenizing Data and Metadata Retrieval in Scientific Applications
}

\author{
Dimitar Misev \\ rasdaman $\mathrm{GmbH}$ \\ Hans-Hermann-Sieling 17 \\ 28759, Bremen, Germany \\ misev@rasdaman.com
}

\author{
Peter Baumann \\ Jacobs University Bremen \\ Campus Ring 1 \\ 28759, Bremen, Germany \\ p.baumann@jacobs-university.de
}

\begin{abstract}
Support for large arrays has been increasingly gaining attention by the database community. Array databases are a quickly expanding category of database management systems that treat large, multidimensional array data as firstclass database citizens, allowing convenient and efficient storage and retrieval. Large array data on its own, however, is rarely meaningful or machine understandable. Therefore, arrays are almost always linked to additional, small metadata, typically managed in relational databases. This leads to an information integration gap, an "impedance mismatch" that we address with the work presented in this paper.

We aim to remedy this with ASQL (Array SQL), an augmentation of the standard relational database language SQL with multidimensional array processing capabilities. Besides core ASQL concepts, this paper presents a general mediator approach to implementing it by combining a relational with an array DBMS. This improves on the most common situation of systems specializing in either relational or array processing, but not both. In particular we implement ASQLDB on top of the array DBMS rasdaman and relational DBMS HSQLDB, and demonstrate its potential for practical applicability through real world use cases. Initial benchmark comparisons against another array DBMS with a similar goal but rather different approach reveal advantageous results for ASQL.
\end{abstract}

\section{INTRODUCTION}

SQL has been lingua franca for any-size data services in business, and has been tremendously successful in delivering flexible, scalable data access technology. Not so, however, in scientific and engineering environments due to the poor or often simply ignored integration of more complex information categories. Large multidimensional grids or "arrays" in particular represent a prevalent data type across most scientific domains, with examples including 1-D sensor data, 2-D satellite images and microscope scans, 3-D x/y/t image timeseries and $\mathrm{x} / \mathrm{y} / \mathrm{z}$ voxel models, as well as $4-\mathrm{D}$ and $5-\mathrm{D}$

Permission to make digital or hard copies of part or all of this work for personal or classroom use is granted without fee provided that copies are not made or distributed for profit or commercial advantage and that copies bear this notice and the full citation on the first page. Copyrights for third-party components of this work must be honored. For all other uses, contact the Owner/Author. Copyright is held by the owner/author(s). DOLAP'15 October 23-23 2015, Melbourne, VIC, Australia ACM 978-1-4503-3785-4/15/10.

http://dx.doi.org/10.1145/2811222.2811223. climate models. Array computational paradigms and model specifically tailored to databases have been published since a while $[2,20,23,19,36,12]$. They have received more significant attention from the database research community only recently, however, as the NoSQL and NewSQL movements have systematically broadened the scope of database models.

While contributing massively to "Big Data", array data is nowadays mostly maintained in ad-hoc solutions crafted by data centers, with functionality often constrained to file download and only gradually increasing functionality portfolios [35, 9, 22, 37, 32]. Even the more progressive users looking at more general solutions are commonly limited to dedicated array databases like rasdaman ${ }^{1}$, SciDB [33], or PostGIS Raster [27]. However, arrays in practice never occur alone, but are ornamented with metadata and embedded in larger overall information structures. Supporting arrays in isolation is thus insufficient when it comes to building modern, complex scientific services and applications. Hence, what is actually missing is native integration with other data types and processing paradigms within the DBMS.

The currently most influential array models are (in order of historical appearance) rasdaman [2, 3], SciQL [38], and SciDB [33]. Notably, ISO SQL:1999 [14] already supports arrays, albeit only very rudimentarily. Arrays are confined there to 1-D only, and without any implicit nor explicit loops for inspecting and manipulating the array elements. It is fair to say that there is no practically useful operational support. Nonetheless, the standard offers a suitable hook for injecting array semantics into its set philosophy.

This is where ASQL (Array SQL) comes in, by extending the relational model with multidimensional arrays. Based on the aforementioned ISO SQL stub it provides a fully-fledged set of structural and operational array constructs completely integrated and compatible with SQL and orthogonal to its set semantics. While based mainly on the conceptualization of rasdaman [3], which has been proven successful in numerous multi-Terabyte operational services $[6,26,7,28,29]$, it also honors recent developments in the array databases field, notably SciDB and SciQL.

Thus, one contribution of this paper is the refinement of the language from its preliminary [25] into its final shape, outlined as a short algebraic background in the next section, followed by a presentation of ASQL in Section 3. An efficient implementation based on the concept of a cross-model mediator is introduced in Section 4. In Section 5 the language is motivated through Earth, Space, and Life science

\footnotetext{
${ }^{1}$ http://rasdaman.org
} 
use cases demonstrating practical feasibility. Related work is discussed in Section 6, and Section 7 concludes the paper.

\section{ARRAY MODEL}

In this section we lay out notation and an algebraic formalization of the array model underpinning ASQL. The array model that we have defined is mostly inspired by the earlier work on Array Algebra [3], an algebra particularly tailored to database query languages. This is further extended with concepts that experience in the meantime has shown are very useful, like dimension names.

Let $[i: k]=\{j \in \mathrm{Z}: i \leq j \leq k\}$ denote the interval of all integers between $i$ and $k$ for some $i \leq k$. An array, then, is a function $a: D \rightarrow V$ which maps points of some $d$-dimensional Euclidean interval

$$
D=\left[l o_{1, n_{1}}: h i_{1, n_{1}}\right] \times \ldots \times\left[l o_{d, n_{d}}: h i_{d, n_{d}}\right] \subset Z^{d}
$$

with $l o_{i, n_{i}} \leq h i_{i, n_{i}}$ and $n_{i} \neq n_{j}$ for all $1 \leq i, j \leq d$, $i \neq j, d>0$, to a non-empty value set $V$. Each vector $x=\left(x_{1, n_{1}}, \ldots, x_{d, n_{d}}\right) \in D$ establishes a coordinate position. The $i$-th dimension is associated with a unique dimension name $n_{i}$ which allows us to, in addition, reference it in a positionally independent manner.

We call a pair $(x, v)$ with $x \in D$ and $v \in V$ a cell with coordinate $x$ and value $v$. The set $D$ of all coordinates of array $a$ is called its (spatial) domain. Thus a $V$ valued multidimensional array $a$ on domain $D$ is of the form:

$$
a=\{(x, a(x)): x \in D, a(x) \in V\}
$$

A distinguishing feature from programming languages is the support for negative integer coordinates. This allows safe array extension in any direction without changing existing values' coordinates, which would invalidate all potential outside references to locations within the array. Further, some arrays naturally have negative indices, such as filter kernels for example.

Non-integer coordinates (real numbers, strings, etc.), however, are not supported. Firstly, such a generalization entails conceptual problems, bringing an unnecessary burden to the model. Handling real-valued coordinates such as geographic positions for example, is substantially more complex in practice involving irregular grids, different Coordinate Reference Systems, etc., and capturing all details is impractical. Secondly, any totally ordered set can be naturally mapped to integers; common practice is to do this by ornamenting the bare arrays appropriately through separate metadata.

\subsection{Domain Operators}

For an array $a$ we define the following probing functions:

- $\operatorname{domain}(a)=D$ returns $a$ 's domain;

- $\operatorname{dimension}(a)=d$ denotes its number of dimensions, a non-negative integer number;

- $l o(a, i)=l o\left(a, n_{i}\right)=l o_{i, n_{i}}$ gives the lowest coordinate of the $i$-th dimension;

- $h i(a, i)=h i\left(a, n_{i}\right)=h i_{i, n_{i}}$ gives the highest coordinate of the $i$-th dimension;

- name $(a, i)=n_{i}$ is the name of the $i$-th dimension;

- $\operatorname{card}(a)=\prod_{i=1}^{d} h i_{i}-l o_{i}+1$ gives its cardinality, or the number of elements as defined by its spatial domain;

\subsection{Value Operators}

An array constructor establishes an array with a specified domain $D$ and an expression $e$ that is evaluated for every coordinate in the domain, thereby producing the array's value set $V$. Dimension names $n_{1}, \ldots, n_{d}$ are bound to the spatial domain for each coordinate position $x$, and can be used in the cell expression $e_{x}$ :

$$
A R R A Y_{D, x}(e)=\left\{\left(x, e_{x}\right): x \in D\right\}
$$

Example. Shift the spatial domain of an array $a$ according to a translation vector $y$ :

$$
A R R A Y_{D+y, x}(a(x-y))
$$

An array condenser aggregates array cell values into a single scalar value. As in the array constructor, dimension names are bound to the spatial domain as iterator variables. Let $\oplus$ be a commutative and associative binary operation with signature $\oplus: T \times T \rightarrow T$, and let $e_{x}$ be an expression of result type $T$ possibly containing occurrences of $x$. Then:

$$
C O N D_{D, x, \oplus}(e)=\oplus_{x \in D} e_{x}=e_{x_{1}} \oplus \cdots \oplus e_{x_{m}}
$$

Example. Compute a sum of the cell values of an array $a$ :

$$
C O N D_{\text {domain }(a), x,+}(a[x])
$$

Both operations resemble a loop over arrays. Loops are at the heart of array processing, but obviously undesirable in database languages as such explicit constructs are unsafe. Therefore, they are implicit in ASQL and other dedicated array languages. Besides the aspect of safe evaluation, this gives room for optimizations in face of partitioned storage as arrays can be traversed in the optimal order [2].

\subsection{Conversion Operators}

Converting an array to a set and vice versa, referred as UNNEST/NEST in the next section, is straightforward. Let $x$ be a vector of elements $x=\left(x_{1, n_{1}}, \ldots, x_{d, n_{d}}\right)$ and $v$ an element, then $::$ is an operation appending $v$ to $x$ :

$$
x:: v=\left(x_{1, n_{1}}, \ldots, x_{d, n_{d}}, v\right)
$$

Basic UNNESTing of the array cell values - without their asssociated coordinates - into an unordered set can be expressed by:

$$
U N N E S T(a)=\{v:(x, v) \in a\}
$$

A set that instead preserves both the cell values and the coordinates would be:

$$
U N N E S T_{\text {ord }}(a)=\{x:: v:(x, v) \in a\}
$$

Converting a set $A$ into an array $a$ is the dual operation of $U N N E S T_{\text {ord }}$, requiring that the set elements are coordinate/cell value tuples:

$$
N E S T(A)=\{(x, v):(x:: v) \in A, d \geq 1\}
$$

\section{ASQL}

Now we can inject the model defined in the previous section into ISO SQL, extending its basic array support to n-D arrays. Data definition is addressed first, then we introduce array operators. 


\subsection{Data Model}

When merging relational and array models a fundamental decision has to be made on the placement of the arrays in the overall model. Most models, including ISO SQL [15], Array Algebra [3], PostGIS Raster [27], SciS̃PARQL [1], introduce arrays as a column type. This allows to see arrays as a plugin to SQL with the overall set model remaining unaffected. We call this approach array-as-attribute. SciQL and SciDB, on the other hand, follow an array-as-table approach where arrays are emulated by tables. Some of the significant drawbacks of array-as-table are that ordinary users must have schema modification rights, which is a security issue; further, the millions of images in a satellite data center will result in millions of "tables", something relational systems are not prepared for; finally, filtering of arrays is generally not possible, considering that SQL does not foresee iteration on table level. Therefore, we follow the former approach as mandated by the ISO SQL standard.

The bracket syntax of programming and scripting languages, which has been adopted by rasdaman, is a commonly known compact notation for array indexing. A shortcoming of such pure positional indexing, however, is that it does not allow to phrase dimension-independent queries like slice this $n$-D cube at time position $T$. Therefore, a time-slicing query on 3-D timeseries needs to look different from slicing a 4 -D climate data cube which is not desirable. To remedy this, dimensions are named, a concept that has been introduced by the OGC Web Coverage Processing Service (WCPS) standard [4], SciQL [38], and SciDB [33].

$\mathrm{SQL}$ comes with rich data type support which is well suited for scientific and engineering applications. We take upon this and let the array cell type be any SQL type, requiring no further changes in the standard. One-dimensional arrays as a collection type are already supported. For practical reasons, and easier maintenance of backwards compatibility, we introduce multidimensional arrays as a separate collection type denoted as MDARRAY. Declaring an MDARRAY requires specifying the array cell type $C T$, dimension names $d n_{i}$ and lower $\left(l o_{i}\right)$ and upper $\left(h i_{i}\right)$ dimension bounds for each dimension $i, 1 \leq i \leq d$ :

$$
C T \text { MDARRAY }\left[d n_{1}\left(l o_{1}: h i_{1}\right), \ldots, d n_{d}\left(l o_{d}: h i_{d}\right)\right]
$$

Dimension bounds are in fact optional, in which case a dimension is unbounded and can extend in $[-\infty,+\infty]$. A special character ' $*$ ' allows to selectively make the lower and/or upper extent unbounded. The list of dimension specifications form the bounding box of an array.

Example. Create a table of Landsat satellite scenes, along with the date when they have been acquired. Each Landsat scene is a 2-D array with dimensions $\mathrm{x}$ and $\mathrm{y}$, and each array element has 5 integer values. The ${ }^{*}$ ' character denotes an unbounded interval, so the $\mathrm{x}$ dimension can be indexed in the interval $[1,5000]$, while y in $[1,+\infty]$.

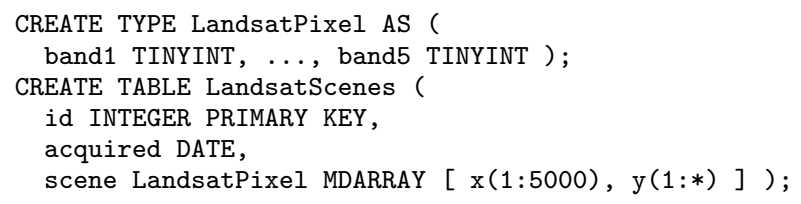

Example. Create a table containing $3 \mathrm{D} x / \mathrm{y} / \mathrm{h}$ floating point temperature data, along with further metadata describing each array cube, like latitude/longitude, resolution, acquisition date. As can be noticed $\mathrm{x}$ and $\mathrm{y}$ do not have dimension restrictions, which automatically makes them unbounded.

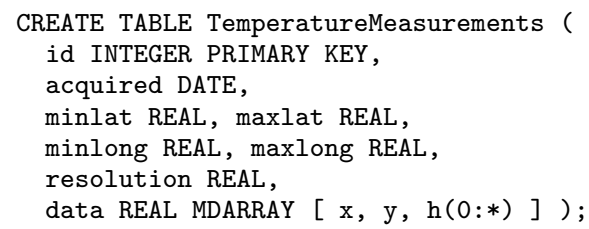

\subsection{Query Language}

\subsubsection{Array Construction}

Constructing a new array is possible in several ways. In direct enumeration, all the array's elements are listed in rowmajor order (unrelated to any internal array representation). The array domain including dimension names and bounds must be specified as well. For example, a 2x2x4 integer array can be enumerated as follows:

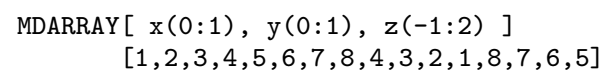

A tabular query result can be converted to an array, as detailed in Section 3.2.6.

Through array iteration: Corresponding to the array constructor introduced in Section 2, each element of the output array is constructed from a value expression following the domain specification. The dimension names can be referenced in the value expression as iterator variables that are bound to every coordinate in the specified domain. For example, the following array iteration expression evaluates to a $2 \times 2 \times 2$ integer array with values $[-1,0,0,1,0,1,1,2]$ :

MDARRAY $[\mathrm{x}(0: 1), \mathrm{y}(0: 1), \mathrm{z}(-1: 0)]$

VALUES $\mathrm{x}+\mathrm{y}+\mathrm{z}$

Finally, an array can be established by reading in contents from a file in some format; this is presented in Section 3.2.7.

\subsubsection{Array Aggregation}

An array aggregation expression returns an aggregated value obtained by iterating over all positions in the spatial domain specified in the OVER clause, evaluating an aggregation expression specified in the USING clause at each position, and combining the result by applying the aggregation operation in the AGGREGATE clause. This operation must be a binary function $f: T \times T \rightarrow T$ defined on the result type $T$ of the aggregation expression, and furthermore commutative and associative, properties that aid in query optimization.

Example. The following expression returns the sum of all values in $A[x(1: 2), y(1: 3)]$ :

AGGREGATE +

OVER $[\mathrm{x}(1: 2), \mathrm{y}(1: 3)]$

USING A[ $\mathrm{x}, \mathrm{y}]$

\subsubsection{Array Inspection Operators}

A few auxiliary operations (cf. Section 2.1) deliver essential information about array objects. Function dimension() returns the number of dimensions of the array; related to this, domain() returns the array's current domain. Function card(), as expected, delivers the number of cells in an array. The array's lower and upper bounds are obtained through functions $l o()$ and $h i()$, resp. Each returns a list of coordinate values, one for each axis; selection of single values is by name or by position. 


\subsubsection{Derived Operators}

Many useful operators can be defined, based on the general array constructor by iteration and array aggregation constructs alone. These operators are not essential, but having them readily available in the query language greatly simplifies the typical array queries. Many of the following examples are on the following array:

$\mathrm{A}[\mathrm{x}(1: 2), \mathrm{y}(0: 3)][[1,2,3,4],[5,6,7,8]]$

Subsetting is an operation that returns an array value consisting of only those elements from its input array A whose positions are in the intersection of the subset domain and the domain of A. Positions in the result which are outside the domain of A are set to NULL. The subset domain is defined as a list of trims and slices, tied to specific dimensions of the array. A trim specifies lower and upper trim bounds for an array's dimension. A wildcard operator provided in the lower and/or upper trim bounds expands to the current bounds of the A. Trimming does not change the dimensionality of the result. E.g, the following expression

$\mathrm{A}[*: *, 1: 2]$

results in a $2-\mathrm{D}$ array with positions $(1,1),(1,2),(2,1),(2,2)$. Note that the previous trim expression is equivalent to

$\mathrm{A}[\mathrm{y}(1: 2)]$

as any missing dimension in a subset operation is automatically set to unbounded in both directions of the dimension. A slice specifies a single point on the selected dimension. Slicing reduces the dimensionality of the result by one, i.e. for $\mathrm{n}$ slices in a subset on $\mathrm{A}$, the dimension $\mathrm{d}$ of the result is given by $\mathrm{d}=\operatorname{dimension}(\mathrm{A})$ - n. For example, the following expression results in a $1-\mathrm{D}$ array with positions $(2,1),(2,2)$, $(2,3)$ :

$A[x(2)]$

Extending is an operation that returns an array with the same elements as the input array, plus additional elements with positions filling up the new, extended result domain with NULLs. The target domain must contain the input array domain. E.g., the following expression returns an array with domain $[\mathrm{x}(0: 10), \mathrm{y}(0: 10)]$ :

extend ( A, [0:10, 0:10])

Shifting returns an array with the same elements as the input array, but with each element shifted by an offset vector. The offset vector must have the same dimensionality as the input array. Example: the following expression returns an array with the same elements, but domain [ -9:-8, 4:7 ]

$\operatorname{shift}(A,[-10,4])$

Scaling is an operation that returns an array with the target domain indicated and values obtained by interpolating the input array element values to the result array domain. The following expression, applied to an array $\mathrm{A}[\mathrm{x}(0: 99)$, $y(0: 99)]$, scales down this array to a domain of $[0: 4$, $0: 4]$ :

scale ( A, [0:4, $0: 4])$

Induced operations return an array with same domain as its input array, where each result cell value is obtained from combining the input cell(s) at the respective position through the operation indicated. This operation must be defined on the input array element type(s), and is defined over them ("induced"). The result array cell type is the result type of the cell operation. The result array dimension is the dimension of the input array(s). In general, any valid operation defined on the input operands, in particular on the individual array elements for the operands which are array values, can be an induced operation.

An induced operation with one array argument is a unary induced operation. For example, the following expression, applied to an array A computes the log of each array cell value; the result type is a FLOAT array:

$\log (\mathrm{A})$

This is equivalent to:

$\operatorname{MDARRAY}[\operatorname{domain}(\mathrm{A})] \log (\mathrm{A}[\mathrm{x}, \mathrm{y}])$

A binary induced operation is an induced operation with two array arguments which both must evaluate to arrays with identical domain. The result is an array computed by applying the operation to each pair of array elements from the operand arrays, based on a corresponding underlying operation on the cell types. If arrays with different cell types are involved the result will be adjusted according to common type expansion rules in programming languages; e.g., float and integer addition will yield a float result. The following expression, applied to an array A and an array B with the same extent, computes the cell-wise sum of both arrays:

$A+B$

This is equivalent to

$\operatorname{MDARRAY}[\mathrm{x}(1: 2), \mathrm{y}(1: 3)] \mathrm{A}[\mathrm{x}, \mathrm{y}]+\mathrm{B}[\mathrm{x}, \mathrm{y}]$

The following expression adds 5 to each element of $\mathrm{A}$

$5+A$

which is equivalent to:

$\operatorname{MDARRAY}[\mathrm{x}(1: 2), \mathrm{y}(1: 3)] 5+\mathrm{A}[\mathrm{x}, \mathrm{y}]$

Finally, from the general aggregation expression, several common array aggregation operations are derived, for adding, averaging, and-ing/or-ing and counting the cells with value $1 /$ TRUE of an array.

\subsubsection{Array Modification}

Arrays can be updated in the regular way through SQL's UPDATE statement. However, there is a special case to be considered. N-D arrays often are built piecewise due to their size; for example, a satellite image timeseries datacube consists of individual images which are ingested one at a time. Therefore, array-valued attributes can be updated in selected regions through a partial update operation. Syntactically, this is achieved by subsetting the update target to indicate the region to be overwritten by the incoming data. Example. Consider modifying an $x / y / t$ cube a in table $T$ with a $2 \mathrm{x} 2$ array at position $(\mathrm{x}, \mathrm{y}, \mathrm{t})=(100,200,3)$ :

UPDATE T

SET T.a $[x(100: 101), y(200: 201), t(3)]=\operatorname{MDARRAY}[[2,3],[4,5]]$

\subsubsection{Conversion between Arrays and Tuple Sets}

Converting an array into a table is useful whenever the perspective of a set of pixels is more adequate. SciQL has an array $\leftrightarrow$ table coercion that converts n-D arrays by associating columns with dimensions, or dimensions with columns. 
ASQL takes on the idea of SciQL, with subtle difference in syntax for consistency with SQL: the existing UNNEST SQL statement is extended to handle the n-D array model, and an explicit NEST is introduced in the array constructor by query for consistency.

UNNESTing an array into a table requires linearizing the array values into a table with each cell in a separate row. When it is desirable to preserve the element's order the WITH ORDINALITY flag can be specified. In this case the result table will in addition have $n=$ dimension(array) integer columns that contain the cell coordinates. Each dimension column is by default named same as the array's dimension name. For example, the below operation

UNNEST (MDARRAY $[\mathrm{x}(1: 2), \mathrm{y}(1: 2)][1,2,5,6]$ )

WITH ORDINALITY AS T(value, $x, y, z$ )

will produce a table $\mathrm{T}$ :

\begin{tabular}{c|c|c|c} 
value & $\mathrm{x}$ & $\mathrm{y}$ \\
\hdashline 1 & & 1 & 1 \\
2 & 1 & 1 \\
5 & 1 & 2 \\
6 & 1 & 2
\end{tabular}

Example. Find the ten most frequent values in an array: This can be done by computing a histogram, which is then converted into to a table in order to get the most frequent values after it's been sorted:

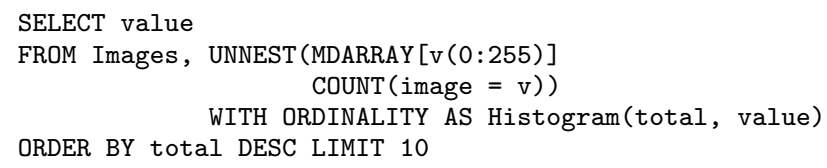

NESTing a table into an array works in a similar way, just reversed. When the table has a single column, then this is converted to an array according to the specified array domain, although no order of the elements is assumed. If the table has additional columns with names that match the array's dimension names, the dimension indices are bound with the values in these columns. Array cells are set to NULL or the DEFAULT value for any coordinate positions defined in the array's domain, but missing from the input table.

Example. The query

ARRAY $[\mathrm{x}, \mathrm{y}]$ NEST (SELECT * FROM T)

produces the array $[1,2,5,6]$. On the other hand

ARRAY $[\mathrm{x}(0: 1), \mathrm{y}]$ NEST (SELECT * FROM T)

sets cells not matched to the indices in T with NULLs:

[NULL , NULL , 5, 6]

\subsubsection{Array Data Format Encoding}

For transmission arrays are encoded in some data format, so format transcoding must be supported. Notably, each such data format comes with its own slate of metadata, ranging from technical details like domain extent to application specific information such as provenance data and georeferencing. While handling of metadata in relational databases is standard today, ASQL adds support for interchange of the data as such.

Array encoding accepts an array and returns a Large Object (LOB), i.e.: a byte string, containing the array encoded in the data format indicated by its MIME type, or some implementation specific format identifier. The format chosen must be able to represent the array domain and element type, otherwise a runtime error is generated. With encode(a, "image/jpeg") the $2 \mathrm{D}$ array a is converted to JPEG, for example.

Array decoding vice versa accepts a LOB, decodes it based on the format identifier provided, and returns an array containing the elements at their proper positions. For example, decode (tiffLOB) converts a TIFF image into an array ready for further storage and processing through ASQL.

\section{IMPLEMENTATION}

The concepts discussed thus far are implemented in an open-source mediator system ASQLDB ${ }^{2}$, which extends the HSQLDB relational DBMS ${ }^{3}$ with support for ASQL. Incoming queries are split into a relational part and array subqueries which are forwarded to some array DBMS for evaluation, in our case rasdaman [30], The results are merged with the results of the pure SQL sub-queries and returned to the client.

Key observation in this approach is that the bulk of the data volume resides and is processed within the array DBMS. This can be leveraged in optimizing mixed query evaluation so that data movement across systems - a typical bottleneck in mediator systems - is minimized. The query translation step from ASQL to the specific array QL, therefore, tries to

- resolve all references to relational data that are mixed with array expressions, as well as

- construct the largest possible array QL sub-queries, so that as much processing as possible per sub-query is pushed down to the ADBMS.

This is the optimal approach, assuming that the relational or mediator systems have no operations applicable to multidimensional arrays beyond the NEST/UNNEST operators.

Example. Let us look at the optimization process through a sample query, "list months with particularly high precipitation in Germany". The bounding coordinates for Germany are fetched from the CountryBoundaries table, and used to compute the pixel coordinates for the subset from the world rainfall data.

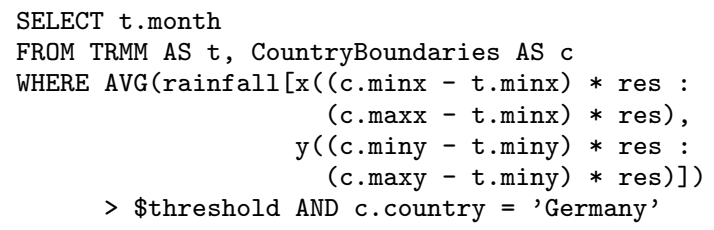

The corresponding query tree on Figure 1 illustrates query optimization. The straightforward but expensive way would be to make two requests to the ADBMS, first the subset on the rainfall data and then the average calculation, as done on the left side. The optimizer instead combines the query tree nodes to form the maximal subtree, which allows to evaluate the average in a single request, and avoid storing intermediate result or shipping whole arrays across the engines.

\footnotetext{
${ }^{2}$ https://github.com/misev/asqldb

${ }^{3}$ http://hsqldb.org/
} 


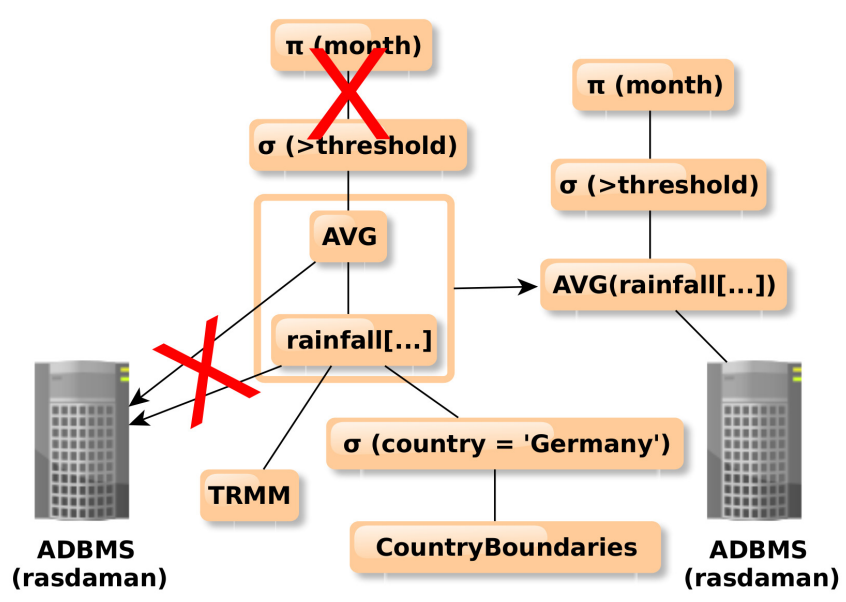

Figure 1: Query tree optimization.

\subsection{Performance Evaluation}

To demonstrate practical feasibility of the implementation we have performed a performance investigation on two representative queries. The benchmark compares performance to SciQL, as this system comes closest to ASQL in terms of SQL integration. Both systems were installed and configured with their default options.

Tests have been run on a standard desktop machine running Debian 7, with an Intel Core i7-3770K CPU, standard 1.8TB 7200 RPM SATA hard disk, and 32GB $1333 \mathrm{MHz}$ RAM. Disk read/write speed was at $134.5 \mathrm{MB} / \mathrm{s}$ and 137 $\mathrm{MB} / \mathrm{s}$, respectively, measured with the following commands:

hdparm -t \$device

dd $b s=1 M$ count=1024 if $=/$ dev/zero of =o conv=fdatasync

The benchmark has been implemented on top of a general array DBMS benchmark [24, 8], publicly available on GitHub ${ }^{4}$. Each query was repeated 5 times; all results outside a $2 \sigma^{5}$ interval of the mean value were discarded and the remaining values averaged. For each query, the DBMS under test got restarted to achieve a cold database. Data size varies logarithmically over $1 \mathrm{~KB}, 100 \mathrm{~KB}, 1 \mathrm{MB}, 100 \mathrm{MB}$, $1 \mathrm{~GB}$ size per object. The following test queries have been run:

- Query 1. The first query corresponds to Example 3 in Section 5.3.1, and computes how close simulated data are to the experimental data (a cost function). The filtering in the WHERE clause has been left out, as this cannot be modeled meaningfully with SciQL.

- Query 2. The Normalized Difference Vegetation Index (NDVI) is a commonly used indicator to assess vegetation cover in remote sensing data. It is computed from the near-infrared and visible red channels, such that in the resulting array vegetation is marked by positive pixels. The test query computes the change of NDVI in successive years.

Table 1 shows the corresponding ASQL and SciQL test queries. Figure 2 shows the benchmark results for both queries. SciQL

\footnotetext{
${ }^{4}$ https://github.com/adbms-benchmark/storage

${ }^{5}$ standard deviation
}

is notably fast on small data sizes, but hits scalability issues as size increases beyond 10-100MB. It took 2680 seconds for SciQL to evaluate the 1 GB size test Query 1, and it failed with an error while evaluating Query 2.

\begin{tabular}{|c|c|c|}
\hline & ASQL & SciQL \\
\hline 1 & $\begin{array}{l}\text { SELECT ABS(SUM(POWER }(z \cdot v- \\
\operatorname{AVG}(z \cdot v), 2)) / \operatorname{CARD}(z \cdot v)- \\
\operatorname{SUM}(\operatorname{POWER}(\mathrm{d} \cdot \mathrm{v}-\operatorname{AVG}(\mathrm{d} \cdot \mathrm{v}), \\
\text { 2)) / (CARD (d.v) - 1) } \\
\text { FROM Dynamic AS d,Zygotic } \\
\text { AS } z\end{array}$ & $\begin{array}{l}\text { SELECT ABS (POWER( STD- } \\
\text { DEV_POP }(\mathrm{z} \cdot \mathrm{v}), 2 \text { ) - POWER( } \\
\text { STDDEV_SAMP }(\mathrm{d} \cdot \mathrm{v}), 2 \text { ) FROM } \\
\text { Dynamic AS d JOIN Zygotic AS } \\
\mathrm{z} \text { ON } \mathrm{z} \cdot \mathrm{x}=\mathrm{d} \cdot \mathrm{x} \text { AND } \mathrm{z} \cdot \mathrm{y}=\mathrm{d} \cdot \mathrm{y}\end{array}$ \\
\hline 2 & $\begin{array}{l}\text { SELECT AVG((a.nir - a.red) } \\
/ \text { (a.nir }+ \text { a.red }))- \\
\text { AVG((b.nir - b.red }) / \\
\text { (b.nir + b.red)) } \\
\text { FROM Landsat09, Landsat10 }\end{array}$ & $\begin{array}{l}\text { SELECT AVG((a.nir - a.red) / } \\
\text { (a.nir + a.red)) - AVG((b.nir } \\
\text { - b.red) / (b.nir + b.red)) } \\
\text { FROM Landsat09 AS a JOIN } \\
\text { Landsat10 AS b ON a.lat }= \\
\text { b.lat AND a.lon = b.lon }\end{array}$ \\
\hline
\end{tabular}

Table 1: Benchmark queries evaluated in ASQLDB and SciQL.

\section{USE CASES}

We proceed with examples from the Earth, Space and Life sciences, to motivate the applicability of ASQL.

\subsection{Earth Sciences}

In [5] fast Web-based map portrayal and navigation based on array queries is presented. For portrayal in a map, one or more layers are superimposed. Visualizing elevation and bathymetry data constitute the computationally most expensive part due to the classification task. In reality, portrayal query gets even more complex as tiles need to be scaled and matched to the request, and overlayed. For example, zooming in on the sea-floor of the Hakon-Mosby underwater mud volcano [16] (Figure 3) results in the query below:

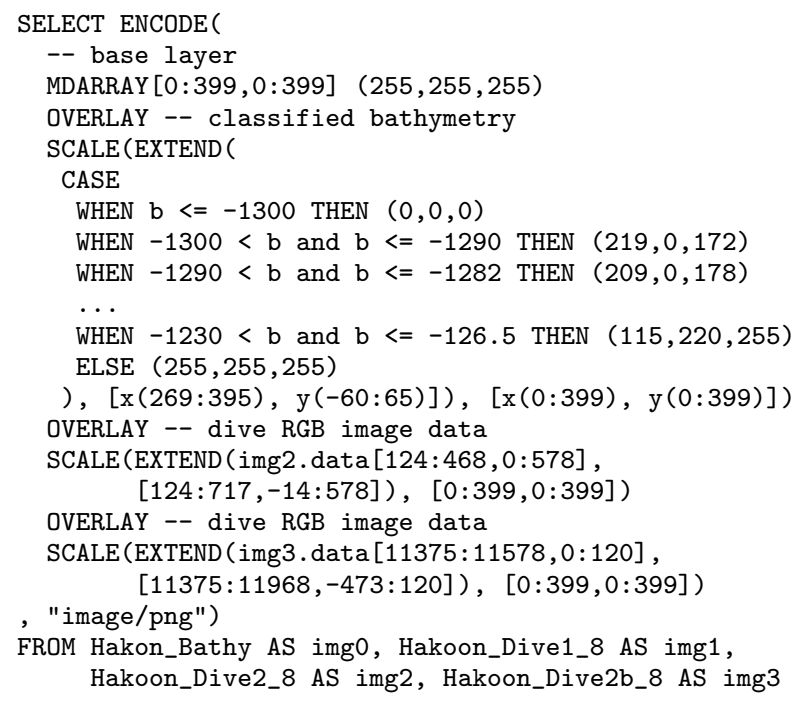

\subsection{Space Sciences}

Astronomy produces massive amounts of array data. The Large Synoptic Survey Telescope (LSST), for example, plans to produce and make publicly accessible petabytes of imagery, catalogs and other metadata [17]. The Transients Key Project (TKP) of LOFAR focuses on building a full 

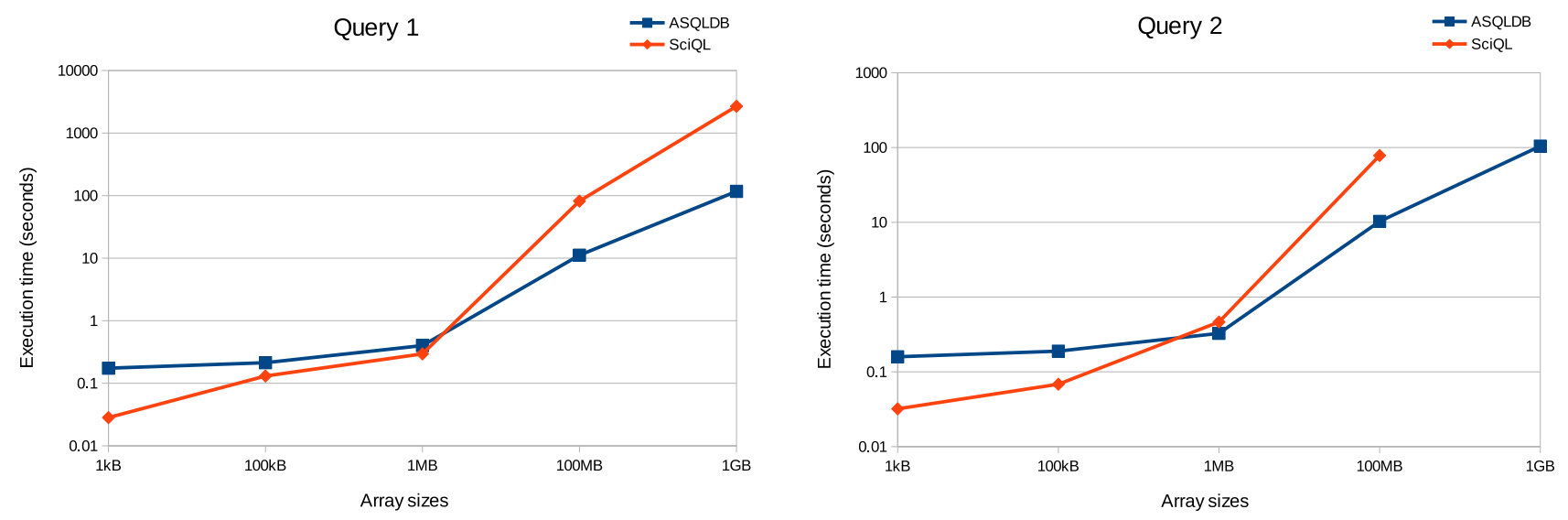

Figure 2: Benchmark results on arrays of different size.

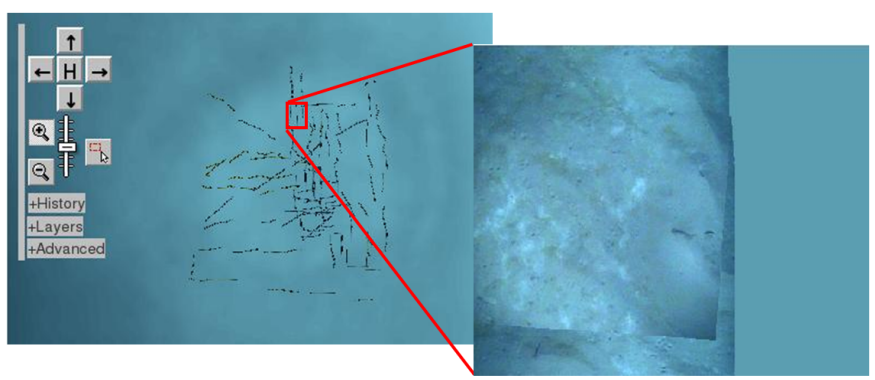

Figure 3: Map service of the Hakon-Mosby underwater mud volcano, showing three seafloor video mosaic layers plus one bathymetry layer [5].

Stokes spectral light-curve database of all detected sources, cross-correlating over multiple catalogues. In [39] the authors examine how an array DBMS (SciQL) could be applied in the context of TKP. The Sloan Digital Sky Survey (SDSS) is creating a 5-wavelength catalog over 10,000 square degrees of the sky, producing 200 million objects in a multi-terabyte database [34]. With rasdaman, flexible retrieval on massive 4-D cosmological simulation datacubes has been demonstrated [18]. Obviously, Space sciences can benefit tremendously from array databases. We therefore believe that data/processing integration as done in SQL/MDA, where image data and metadata coexist next to each other, can be expected to substantially reduce query complexity and response times of such scientific workloads.

\subsection{Life Sciences}

\subsubsection{Gene expression data management}

The management, retrieval and analysis of multidimensional gene expression data [29] lands itself nicely to the integration of relational and array data. Data ranges from two-dimensional images of gene expression patterns to complex arrays describing spatio-temporal dynamics of gene expression within regulatory network. Embryo, gene, simulation and further metadata is stored as relational data.

Example 1. Extract regions where the level of gene expression is below, equal or exceeding a predefined \$threshold

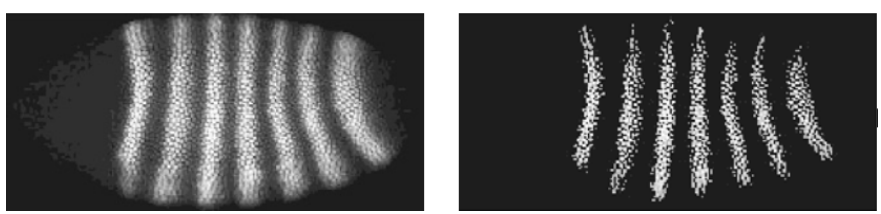

Figure 4: Threshold filtering of Drosophila gene expression activity (left: original slice, right: filtered slice) [29].

(Figure 4). The embryo is selected by $\$$ name.

SELECT e.image $[z(\$ c h)] *$ (e.image $(z(\$ c h)]>$ \$threshold)

FROM Embryolmages AS e, embryo AS m

WHERE e.id $=$ m.id AND e.name = '\$name'

Example 2. Combine slices at different channels of the confocal microscope into an RGB image. Data from the first channel is mapped to the red color, second component to green and third to blue (Figure 5).

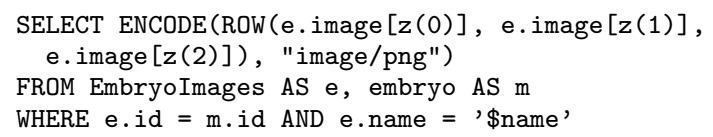

Example 3. The cost function is a measure of how close simulated data are to the experimental data, computed as the sum of squared deviations of computed gene expression level from the experimentally observed level. Parameter values used in simulation data generation are sequentially varied in order to reach a global minimum of the cost function. The query below computes the cost function for data at a specific \$time point after egg deposition in seconds.

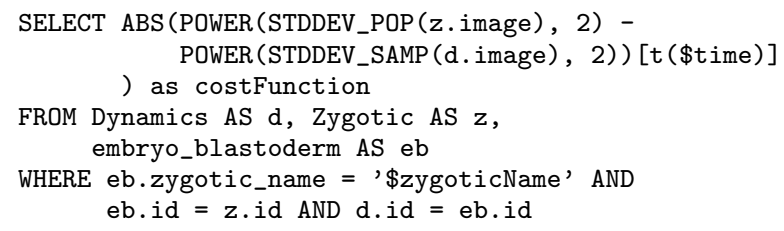

Example 4. Gene expression pattern images can be superimposed with a binary nuclei mask to isolate nuclei. Quantitative data like nuclei location within the embryo and their average intensity can then be determined. 


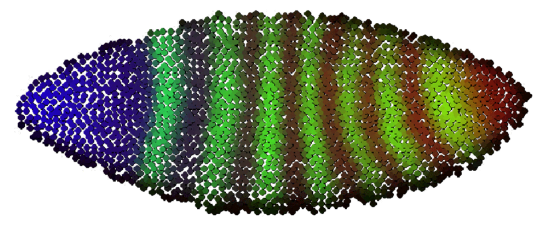

Figure 5: Combination of different channels into an RGB image [29].

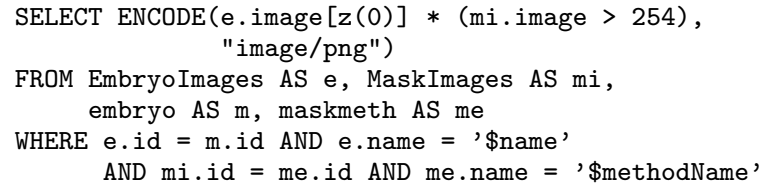

\subsubsection{Human brain imaging}

A major goal of neuroscience nowadays is to understand how functional activations in the brain relate to its microstructure, and to what extent can consistency in the description of function contributions of cortical activated fields be achieved. A task like this can only be tackled on a global level, as has been attempted already with the European Computerized Human Brain Database (ECHBD) [13] and subsequently in the NeuroGenerator project [31].

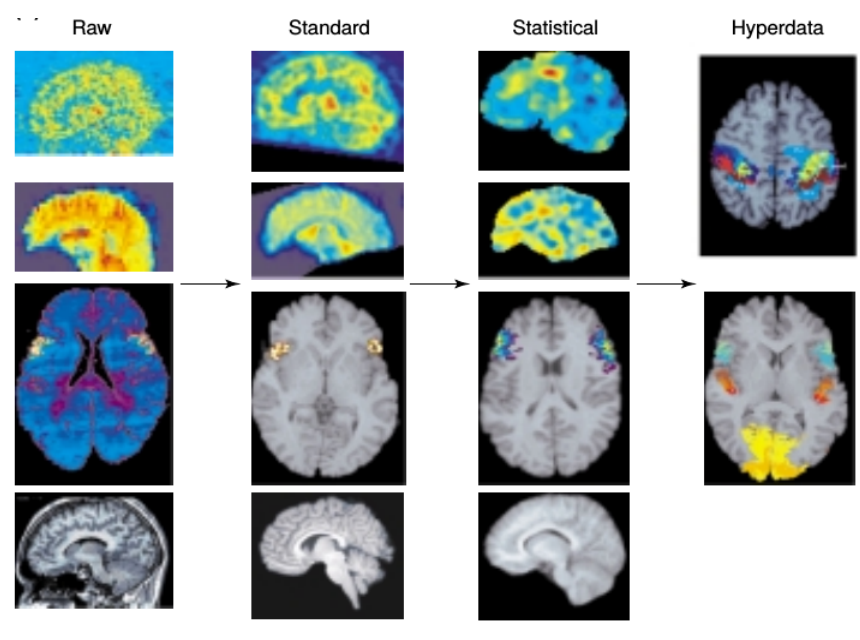

Figure 6: Brain data processing in the NeuroGenerator project $[31]$.

Example. Retrieve a parasagittal view (slice position \$pos provided as input by the user) of all images, in which critical activations appear in the Hippocampus, encoded in TIFF format. The percentage of the masked hippocampus area that should be critically activated for an image to be considered is given by the user as $\$$ threshold $\in(0,1]$. Figure 6 shows examples of the the image data.

SELECT ENCODE (f.image [ $z$ (\$pos)], "image/tiff")

FROM FMRIImage AS $f$, PETImage as $p$, Mask AS $m$,

WHERE SubjectImages AS si, ImageMasks AS im

m.id = im.maskId AND m.region = 'hippocampus'

AND im.subjectId = si.subjectId AND

si.imageId $=f . i d$ AND si.imageId $=$ p.id AND

count_cells ( p.image > 227 AND m.binaryMask )

/ count_cells ( m.binaryMask ) > \$threshold

\section{STATE OF THE ART}

Several approaches for array handling in a database context have been proposed; the most advanced projects (in order of historical appearance) are rasdaman [3], PostGIS Raster [27], SciQL [38], SciDB [33], and most recently SciSPARQL [1] and EXTASCID [10, 11].

An attempt to achieve integration of different array models has been started, published in a blog [21]. Architects of rasdaman, SciQL, and SciDB convened to merge experience in array handling and establish a common algebraic framework. A list of desirable operations has been collected, but not yet agreed; for example, no set of minimal operations, comparable to Array Algebra, has been derived yet. The paper itself points out that it is a preliminary version and that further work is required.

SciQL extends SQL with multidimensional array capabilities. The array-as-table approach, however, is problematic, as previously discussed. It is unclear how array-as-table scales to millions of arrays, such as with large satellite image archives, given that SQL does not foresee iteration over table sets, so that finding and filtering the data of interest in a standard way is not possible. Furthermore, SciQL has allowed dimensions of any predefined type with arbitrary resolution. We consider this an unnecessary burden to the model, without gaining expressive power: on conceptual level, a mapping of arbitrary regular or even irregular coordinates to contiguous integer coordinates is always possible, which has been proven by large-scale geo services ${ }^{6}$. Our goal is to specify a simple and robust, yet comprehensive core model, and other dimension types lead to associative arrays rather than the classical array concept we strive for here. Finally, SciQL currently is described only in terms of examples, but has no underlying formal semantics.

An approach similar to SciQL is pursued by SciDB, designed as a "pure" Array DBMS. The APPLY operation maps to specific individual operations. In its implementation, SciDB heavily relies on UDF (user-defined function) technology for the implementation of operations. The difference to ASQL is that APPLY is modeled as a black box outside of the semantic definition creating a "semantic leak" which becomes visible once complexity considerations and assumptions on the functions cell access behavior are considered. ASQL, on the other hand, establishes a clear semantics by relying on Array Algebra. This underlines that arrays as second-order concepts require tight integration for a smooth, user-friendly array embedding, which cannot be obtained by (first-order) UDFs.

PostGIS Raster [27] is a library using PostgreSQL objectrelational extensibility feature, hence is also relying on UDFs. Queries are of limited flexibility, the resulting UDF syntax is not particularly intuitive, and does not seamlessly integrate set and array expressions. Again, a formal underpinning is lacking - the model is described through manual and code only. Finally, PostGIS Raster is tuned towards geo applications while ASQL strives to be domain-neutral.

Several SciQL and SciDB concepts have been adopted, though. Named dimensions provide progress over Array Algebra, as dimensions of no interest can be left out in a subsetting whereas Array Algebra requires all dimensions to be present. This increases expressiveness as knowledge of the array's dimensionality is not necessary anymore. Further,

\footnotetext{
${ }^{6}$ http://earthserver.eu/
} 
UNNEST and NEST operators as known from SciQL (albeit in different syntax) allow smooth transformation from arrays to tables and back.

\section{CONCLUSION}

Array data is at the heart of manifold science and engineering domains. It is generally accepted today, therefore, that arrays have to become integral part of the overall data type orchestration in information systems. The ASQL model proposed achieves a tight, seamless, orthogonal integration into the relational model, extending its applicability to a large class of "Big Data" challenges. Its formal algebraic framework provides a solid underlying semantics definition based on a minimal operation set. The ASQL data model extends SQL's array stub from 1-D to n-D arrays on arbitrary cell types. In keeping with the SQL philosophy, arrays remain collection types related to, e.g., multisets. In terms of operations, ASQL smoothly extends the rudimentary array support with generalized, declarative and $\mathrm{nD}$-applicable operations. Aside from ISO SQL, ASQL duly takes into account - and actually is based on - the collective experience of the array database research community, integrating relevant work done in rasdaman, SciQL, SciDB and, implicitly, further related models like AQL and AML.

This paper's technical contribution consists of a comprehensive presentation of ASQL with its unifying array/set integration, pointing out implementability through a mediatorbased architecture and exemplary cross-model optimization, underpinning this with performance measurements, and demonstrating feasibility through a broad set of applications. A core objective of this contribution is to present the forthcoming SQL/MDA standard to the database community to give sufficient opportunity for discussion and critique prior to the final release of the standard. This way we want to ensure that the scientifically and technologically best concepts drive the forthcoming SQL/MDA standard.

From the observations emerging in this work we actually propose new research on mediators. While they traditionally have served to integrate different relational flavours, now the challenge is to establish a common framework for crossmodel integration, such as set, tree, graph, array, and document models. This leads to new challenges in conceptual modelling, architectures, and optimization.

\section{ACKNOWLEDGEMENTS}

This research has been co-funded by the European Commission through the FP7 EarthServer and H2020 EarthServer2 projects.

\section{REFERENCES}

[1] A. Andrejev and T. Risch. Scientific SPARQL: Semantic Web Queries over Scientific Data. In Proc. IEEE 28th Intl. Conference on Data Engineering Workshops, ICDEW '12, pages 5-10. IEEE Computer Society, 2012.

[2] P. Baumann. Management of Multidimensional Discrete Data. VLDB Journal, 3(4):401-444, Oct. 1994.

[3] P. Baumann. A Database Array Algebra for Spatio-Temporal Data and Beyond. In Proc. 4th Intl. Workshop on Next Generation Information
Technologies and Systems, NGITS '99, pages 76-93, London, UK, 1999. Springer-Verlag.

[4] P. Baumann. OGC Web Coverage Processing Service (WCPS) Language Interface Standard. $O G C$ 08-068r2, 2009.

[5] P. Baumann, C. Jucovschi, and S. Stancu-Mara. Efficient Map Portrayal Using a General-Purpose Query Language. In Proc. 20th Intl. Conference on Database and Expert Systems Applications, DEXA '09, pages 153-163, 2009.

[6] P. Baumann, P. Mazzetti, J. Ungar, R. Barbera, D. Barboni, A. Beccati, L. Bigagli, E. Boldrini, R. Bruno, A. Calanducci, P. Campalani, O. Clement, A. Dumitru, M. Grant, P. Herzig, K. Kakaletris, L. Laxton, P. Koltsida, K. Lipskoch, A. Mahdiraji, S. Mantovani, V. Merticariu, A. Messina, D. Misev, S. Natali, S. Nativi, J. Oosthoek, J. Passmore, M. Pappalardo, A. Rossi, F. Rundo, M. Sen, V. Sorbera, D. Sullivan, M. Torrisi, L. Trovato, M. Veratelli, and S. Wagner. Big data analytics for earth sciences: the earthserver approach. International Journal of Digital Earth, 2015.

[7] P. Baumann and D. Misev. Towards Scalable Ad-Hoc Climate Anomalies Search. In Proc. ACM SIGSPATIAL International Workshop on Analytics for Big Geospatial Data, BigSpatial '12, pages 101-110, New York, NY, USA, 2012. ACM.

[8] P. Baumann and H. Stamerjohanns. Benchmarking Large Arrays in Databases. In Proc. Workshop on Big Data Benchmarking, pages 94-102, December 2012.

[9] J. Blower, A. Gemmell, G. Griffiths, K. Haines, A. Santokhee, and X. Yang. A Web Map Service implementation for the visualization of multi-dimensional gridded environmental data. Environmental Modelling \& Software, 47(0):218 - 224, 2013.

[10] Y. Cheng and F. Rusu. Astronomical Data Processing in EXTASCID. In Proc. 25th International Conference on Scientific and Statistical Database Management, pages 47:1-47:4. ACM, 2013.

[11] Y. Cheng and F. Rusu. Formal Representation of the SS-DB Benchmark and Experimental Evaluation in EXTASCID. Distributed and Parallel Databases, pages 1-41, 2013.

[12] R. Cornacchia, S. Héman, M. Zukowski, A. P. Vries, and P. Boncz. Flexible and Efficient IR Using Array Databases. VLDB Journal, 17(1):151-168, 2008.

[13] J. Fredriksson, P. Roland, and P. Svensson. Rationale and design of the European Computerized Human Brain Database System. In Eleventh International Conference on Scientific and Statistical Database Management, pages 148-157, Aug 1999.

[14] ISO. Information Technology - Database Language SQL. Standard No. ISO/IEC 9075:1999, International Organization for Standardization (ISO), 1999.

[15] ISO. ISO/IEC 9075-1:2003: Information technology - Database languages - SQL - Part 1: Framework (SQL/Framework). ISO, Geneva, Switzerland, 2003.

[16] K. Jerosch. Identification of seafloor provinces specific applications at the deep-sea Hakon Mosby Mud Volcano and the North Sea. Phd thesis, Bremen University, 2006. 
[17] J. Kantor, T. Axelrod, J. Becla, K. Cook, S. Nikolaev, J. Gray, R. Plante, M. Nieto-Santisteban, A. Szalay, and A. Thakar. Designing for Peta-Scale in the LSST Database. In Astronomical Data Analysis Software and Systems XVI ASP Conference Series, page 3, 2006.

[18] K. Kleese and P. Baumann. Intelligent Support for High I/O Requirements of Leading Edge Scientific Codes on High-End Computing Systems - The ESTEDI Project. In Proc. Sixth European SGI/Cray MPP Workshop, 7-8 September 2000.

[19] A. Lerner and D. Shasha. AQuery: query language for ordered data, optimization techniques, and experiments. In Proc. 29th Intl. Conf. on Very Large Data Bases, VLDB '03, pages 345-356, 2003.

[20] L. Libkin, R. Machlin, and L. Wong. A Query Language for Multidimensional Arrays: Design, Implementation, and Optimization Techniques. In Proc. ACM SIGMOD International Conference on Management of Data, SIGMOD '96, pages 228-239, New York, NY, USA, 1996. ACM.

[21] D. Maier, P. Baumann, M. Kersten, K.-T. Lim, and M. Stonebraker. ArrayQL Algebra: version 3. Seen: 2015-feb-22, http://www.xldb.org/wp-content /uploads/2012/09/ArrayQL_Algebra_v3+.pdf, 2012.

[22] P. P. Mak, J. Blower, J. Caron, E. Davis, A. Santokhee, and N. Bindoff. Integrating ncWMS into the THREDDS Data Server. 2009.

[23] A. P. Marathe and K. Salem. Query Processing Techniques for Arrays. VLDB Journal, 11(1):68-91, Aug. 2002.

[24] G. Merticariu, D. Misev, and P. Baumann. Towards a General Array Database Benchmark: Measuring Storage Access. Submitted for publication, 2015.

[25] D. Misev and P. Baumann. Extending the SQL Array Concept to Support Scientific Analytics. In Conference on Scientific and Statistical Database Management, SSDBM '14, Aalborg, Denmark, June 2014.

[26] D. Misev, P. Baumann, and J. Seib. Towards Large-Scale Meteorological Data Services: A Case Study. Datenbank-Spektrum, 12(3):183-192, 2012.

[27] R. Obe and L. Hsu. PostGIS in Action. Manning Pubs., 2011.

[28] J. Oosthoek, J. Flahaut, A. Rossi, P. Baumann, D. Misev, P. Campalani, and V. Unnithan. PlanetServer: Innovative approaches for the online analysis of hyperspectral satellite data from Mars. Advances in Space Research, 53(12):1858-1871, 2014.

[29] A. Pisarev, E. Poustelnikova, M. Samsonova, and P. Baumann. Mooshka: a system for the management of multidimensional gene expression data in situ. Information Systems, 28(4):269-285, June 2003.

[30] rasdaman Gmbh. rasdaman Query Language Guide, 9.1 edition, 2015.

[31] P. Roland, G. Svensson, T. Lindeberg, T. Risch, P. Baumann, A. Dehmel, J. Frederiksson, H. Halldorson, L. Forsberg, J. Young, and K. Zilles. A database generator for human brain imaging. Trends in Neurosciences, 24(10):562-564, Oct. 2001.

[32] Y. Sagarminaga, I. Galparsoro, R. Reig, and J. A. Sánchez. Development of ITSASGIS-5D: seeking interoperability between Marine GIS layers and scientific multidimensional data using open source tools and OGC services for multidisciplinary research. In A. Abbasi and N. Giesen, editors, EGU General Assembly Conference Abstracts, volume 14, apr 2012.

[33] M. Stonebraker, P. Brown, A. Poliakov, and S. Raman. The Architecture of SciDB. In Proceedings of the 23rd International Conference on Scientific and Statistical Database Management, SSDBM'11, pages 1-16, Berlin, Heidelberg, 2011. Springer-Verlag.

[34] A. S. Szalay, P. Z. Kunszt, A. Thakar, J. Gray, D. Slutz, and R. J. Brunner. Designing and Mining Multi-terabyte Astronomy Archives: The Sloan Digital Sky Survey. In Proceedings of the 2000 ACM SIGMOD International Conference on Management of Data, SIGMOD '00, pages 451-462, New York, NY, USA, 2000. ACM.

[35] Unidata. Thredds data server (tds). www.unidata.ucar.edu/software/thredds/tds/. Accessed online on 2015-feb-22.

[36] A. R. van Ballegooij. RAM: a Multidimensional Array DBMS. In Proceedings of the 2004 international conference on Current Trends in Database Technology, EDBT'04, pages 154-165. Springer-Verlag, 2004.

[37] N. A. Walton, E. Gonzalez-Solarez, S. Dalla, A. Richards, and J. Tedds. AstroGrid: A place for your science. Astronomy \& Geophysics, 47(3):3.22-3.24, 2006.

[38] Y. Zhang, M. L. Kersten, M. Ivanova, and N. Nes. SciQL, Bridging the Gap between Science and Relational DBMS. In IDEAS, pages 124-133. ACM, 2011.

[39] Y. Zhang, L. H. A. Scheers, M. L. Kersten, M. Ivanova, and N. J. Nes. Astronomical Data Processing Using SciQL, an SQL Based Query Language for Array Data. In Astronomical Data Analysis Software and Systems, 2011. 